\title{
II. Ueber die
}

Condensation von Hydantoïn mit Formaldehyd; von Robert Behrend und Rudolf Niemeyer. ${ }^{1)}$

\section{IIferza TafeI I.}

Die folgenden Versuche wurden in der Absicht angestellt, eine bequeme Methode zur Darstellung von $\beta$-Methylhydantoïn auszuarbeiten. Wie die Harnsäure ${ }^{2}$ ) lässt sich nun zwar das Hydantoïn mit Formaldehyd leicht $\mathrm{zu}$ einer Oxymethylenverbindung vereinigen

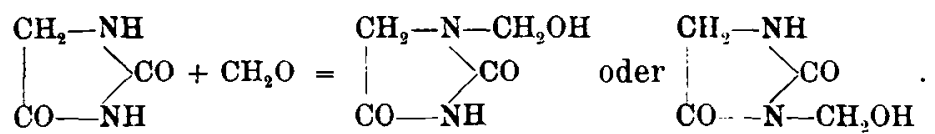

Alle Versuche jedoch, diese zum Methylhydantoïn zu reduciren, eine Reaction, welche bei der Oxymethylharnsäure sich leicht und glatt durchführen lässt ${ }^{3}$ ), scheiterten an der Neigung des Oxymethylhydantoïns, in complicirtere condensationsproducte überzugehen.

Durch die verschiedensten reducirenden Mittel, die in saurer oder alkalischer Lösung zur Verwendung kamen, wurde der Körper entweder nicht angegriffen oder es entstanden die weiter unten beschriebenen Condensationsproducte. Gelegentlich wurde auch Hydantoïn zurückgebildet. Zur Umgehung dieser Schwierigkeit wurde versucht, das Oxymethylhydantoïn zunächst in

1) Vergl. R. Niemeyer, Inauguraldissertation, Hannover 1908.

2) D. R. P. 102158.

8. R. P. 105345. 
Fischer und Niemeyer, Ueber die Condensation etc. 39

Chlormethylhydantoïn überzuführen und dieses dann zu reduciren.

Das Chlormethylhydantoïn lässt sich mit Hülfe von Phosphorpentachlorid und schon von conc. Salzsäure sehr leicht darstellen.

$$
\mathrm{C}_{8} \mathrm{H}_{9} \mathrm{~N}_{2} \mathrm{O}_{2} \cdot \mathrm{CH}_{2} \mathrm{OH}+\mathrm{HCl}=\mathrm{C}_{8} \mathrm{H}_{3} \mathrm{~N}_{2} \mathrm{O}_{2} \cdot \mathrm{CH}_{2} \mathrm{Cl}+\mathrm{H}_{2} \mathrm{O} \text {. }
$$

Aber ebenso leicht, wie das Hydroxyl sich gegen Chlor austauschen lässt, wird das Chlor auch wieder durch Hydroxyl ersetzt und bei den Reductionsversuchen entstehen daher dieselben Producte wie aus dem Oxymethylhydantoïn.

Ob das Oxymethyl in $\alpha$-oder $\beta$-Stellung in das Hydantoïn eintritt, konnte unter diesen Umständen nicht ermittelt werden; um die Constitution der Condensationsproducte, wenigstens soweit es zur Zeit möglich ist, durch Formelbilder veranschaulichen zu können, wurde angenommen, dass der Eintritt zuerst in $\beta$-Stellung erfolgt.

Das zu den Versuchen nöthige Hydantoïn wurde nach dem Verfahren von Siemonsen dargestellt.

\section{Oxymethylhydantoïn,$$
\mathrm{C}_{4} \mathrm{H}_{8} \mathrm{~N}_{2} \mathrm{O}_{\mathbf{a}} \text {. }
$$

$10 \mathrm{~g}$ Hydantoïn werden in $8,5 \mathrm{~g}$ käuflicher $40 \%$ iger Formaldehydlösung, entsprechend etwa $1 \frac{1}{3}$ Mol. Formaldehyd auf 1 Mol. Hydantoïn in der Hitze gelöst. Beim Erkalten erstarrt die Lösung zu einem festen Filz von feinen weissen Nädelchen. Man saugt scharf $a b$ und wäscht mit Alkohol und Aether aus. Die Ausbeute beträgt $7,2-9,9 \mathrm{~g}$, im Mittel $8,1 \mathrm{~g}$; aus den eingeengten Mutterlaugen krystallisiren innerhalb 1-2 Tagen noch 1,5-2,2 g. Die Gesammtausbeute beträgt also im Mittel $9,9 \mathrm{~g}$ oder $76,5 \%$ der berechneten. Der Rest stellt einen dicken Syrup dar, der sich allmählich mit Krystallen durchsetzt; doch lassen sich diese nicht mehr absaugen.

4) Diese Annalen 393, 109 (1904). 
In Wasser ist der Körper sehr leicht löslich; wenn man längeres Kochen vermeidet, kann man ihn daraus in spiessartigen Prismen krystallisirt erhalten. Besser verwendet man zum Umkrystallisiren Alkohol, in dem er ziemlich schwer löslich ist; er kommt daraus in verfilzten Nädelchen. Der Körper schmilzt unscharf zwischen $125^{\circ}$ und $135^{\circ}$; erhitzt man ihn einige Stunden auf $120-130^{\circ}$, so bildet sich eine glasklare Schmelze, aus deren Lösung in heissem Wasser die weiter unten beschriebene Verbindung $\mathrm{C}_{7} \mathrm{H}_{8} \mathrm{~N}_{4} \mathrm{O}_{4}$ krystallisirt. Bei längerem Erhitzen trübt sich die Masse wieder, wird hart und hygroscopisch und lässt sich nun aus Wasser nur noch als Syrup abscheiden.

Beim Kochen des Oxymethylhydantoïns mit Wasser wird langsam Formaldehyd abgespalten; die nach halbstündigem Kochen auskrystallisirte Substanz schmolz bei $80-100^{\circ}$, nach einstündigem Kochen schied sich reines Hydantoïn vom Schmelzp. $216^{\circ}$ ab.

Analyse des aus Wasser umkrystallisirten Oxymethylhydantoïns:

I. $0,1681 \mathrm{~g}$ gaben $0,2292 \mathrm{CO}_{2}$ und $0,0792 \mathrm{H}_{8} \mathrm{O}$.

II. $0,2065 \mathrm{~g} \quad, \quad 0,2790 \mathrm{CO}_{2} \quad, \quad 0,0812 \mathrm{H}_{2} \mathrm{O}$.

III. $0,2393 \mathrm{~g} \quad " \quad 46$ ccm feuchtes Stickgas bei $14^{\circ}$ und $737 \mathrm{~mm}$

Druck.

IV. $0,3062 \mathrm{~g} \quad, \quad 55,9 \mathrm{ccm} \quad, \quad, \quad, 10^{\circ}, 763 \mathrm{~mm}$ Druck.

\begin{tabular}{lrcrrr} 
& \multicolumn{2}{c}{ Ber. für } & \multicolumn{4}{c}{ Gef. } \\
& $\mathrm{C}_{4} \mathrm{H}_{6} \mathrm{~N}_{2} \mathrm{O}_{8}$ & I & II & III & IV \\
$\mathrm{C}$ & 36,92 & 37,19 & 36,85 & - & - \\
$\mathrm{H}$ & 4,61 & 5,23 & 4,37 & - & - \\
$\mathrm{N}$ & 21,54 & - & - & 21,81 & 21,85
\end{tabular}

Chlormethylhydantoïn,<smiles>O=C1CN(CCl)C(=O)N1</smiles>

5 g Oxymethylhydantoïn werden mit überschüssigem fein zerriebenem Phosphorpentachlorid in einem mit Chlor- 
calciumrohr verschlossenen Kolben gut gemischt. Nach einiger Zeit tritt Erwärmung und lebhafte Salzsäureentwickelung ein, nach deren Beendigung man unter öfterem Umrühren 24 Stunden stehen lässt und schliesslich 1 Stunde auf $55-60^{\circ}$ erwärmt, bis kein Chlorwasserstoff mehr entweicht. Der von Krystallen durchsetzte dünnflüssige Syrup wird entweder durch Ausziehen mit Benzol von den Phosphorchloriden befreit oder mit absolutem Alkohol behandelt. In letzterem Falle ist aber Vorsicht und gute Kühlung nöthig, da warmer Alkohol das Chlormethylhydantoin zersetzt.

Die zurückbleibenden Krystalle werden mit wenig Alkohol und dann mit Aether gewaschen und im Exsiccator getrocknet. Die Ausbeute beträgt im Mittel $3,7 \mathrm{~g}$ oder $65 \%$ der berechneten Menge. An feuchter Luft zersetzt sich der Körper allmählich unter Abgabe von Salzsäure, beim Frwärmen mit Wasser oder Alkohol wird alles Chlor als Salzsäure abgespalten. Zum Umkrystallisiren kann man Benzol verwenden, in welchem er allerdings recht schwer löslich ist, oder Chloroform, welches inn leichter aufnimmt. Er krystallisirt in kleinen gut ausgebildeten Prismen; der Schmelzpunkt liegt unscharf zwischen 150 und $157^{\circ}$. Zur Chlorbestimmung wurde die Substanz in heissem verdünnten Ammoniak gelöst und das Chlor als Chlorsilber oder titrimetrisch bestimmt. Verschiedene Rohproducte enthielten 22,65̃, 22,70 und $20,80 \%$ Chlor.

Analỵse des aus Chloroform krystallisirten Productes:

$0,1640 \mathrm{~g}$ gaben $0,1908 \mathrm{CO}_{2}$ und $0,0413 \mathrm{H}_{2} \mathrm{O}$.

$0,1976 \mathrm{~g} \quad 33,5 \mathrm{~cm}$ feuchtes Stickgas bei $10^{\circ}$ und $749 \mathrm{~mm}$

Druck.

$0,2033 \mathrm{~g}$ verbrauchten $27,8 \mathrm{ccm} \mathrm{N} / 20 \mathrm{Ag} \mathrm{NO} \mathrm{N}_{8}$-I

$\begin{array}{lcr} & \text { Ber. für } \mathrm{C}_{4} \mathrm{H}_{8} \mathrm{~N}_{2} \mathrm{O}_{2} \mathrm{Cl} & \text { Gef. } \\ \mathrm{C} & 32,32 & 31,73 \\ \mathrm{H} & 3,40 & 2,79 \\ \mathrm{~N} & 18,85 & 19,91 \\ \mathrm{Cl} & 23,87 & 24,20\end{array}$


Viel einfacher erhält man das Chlormethylhydantoïn, indem man Oxymethylhydantoin aus conc. Salzsäure umkrystallisirt :

$$
\mathrm{C}_{4} \mathrm{H}_{6} \mathrm{~N}_{8} \mathrm{O}_{3}+\mathrm{HCl}=\mathrm{C}_{4} \mathrm{H}_{8} \mathrm{~N}_{2} \mathrm{O}_{2} \mathrm{Cl}+\mathrm{H}_{3} \mathrm{O} \text {. }
$$

$5 \mathrm{~g}$ Oxymethylhydantoïn wurden in $8.5 \mathrm{~g}$ heisser $36 \%$ iger Salzsäure gelöst. Nach 24 Stunden wurden die ausgeschiedenen Krystalle abgesaugt, mit möglichst wenig kaltem Wasser gedeckt, mit wenig Alkohol und dann mit Aether gewaschen. Aus der Mutterlauge schieden sich nach 2 Tagen noch weitere Mengen ab. Die Gesammtausbeute bei verschiedenen Versuchen betrug im Mittel $4,3 \mathrm{~g}$ oder $75 \%$ der berechneten. Das Product erwies sich als identisch mit dem oben beschriebenen.

Durch Condensation von Hydantoïn mit Formaldehyd bei Gegenwart von Salzsäure entstehen complicirtere C'ondensationsproducte.

$$
\text { Körper } \mathrm{C}_{8} \mathrm{H}_{10} \mathrm{~N}_{4} \mathrm{O}_{5} \text {. }
$$

Für die Darstellung der Verbindung $\mathrm{C}_{8} \mathrm{H}_{10} \mathrm{~N}_{4} \mathrm{O}_{5}$ hat sich bei einer grösseren Reihe von Versuchen folgendes Verfahren als das zweckmässigste ergeben.

$5 \mathrm{~g}$ Hydantoïn werden in $4,5 \mathrm{~g}$ heisser $40 \%$ iger Formalinlösung unter Zusatz von zwei Tropfen conc. Salzsäure gelöst und die Lösung in einer Schale sechs Tage lang der Krystallisation überlassen. Die zu einer larten, meist fest am Boden der Schale haftenden Kruste vereinigten Krystalle werden zerrieben und mit möglichst wenig Wasser bis zum Verschwinden der sauren Reaction gewaschen. Zur Beschleunigung des Trocknens können sie mit Alkohol und Aether gewaschen werden. Ihr Gewicht beträgt im Mittel 5,6 g oder $92 \%$ der berechneten Menge. Der Körper lässt sich aus etwa der fünffachen Menge heissen Wassers umkrystallisiren, er verträgt auch längeres Kochen mit reinem Wasser, sind aber wenn auch nur geringe Mengen von Salzsäure vorhanden, so geht er unter tbspaltung von Formaldehyd 
in den Körper $\mathrm{C}_{7} \mathrm{H}_{8} \mathrm{~N}_{4} \mathrm{O}_{4}$ über. Beim Umkrystallisiren geht stets viel verloren, man erhält nur etwa $50 \%$ der berechneten Ausbente an reinem umkrystallisirten Product. Die Krystalle stellen derbe farblose Prismen dar. In Alkohol sind sie sehr schwer löslich, ihr Schmelzpunkt liegt je nach der Art des Erhitzens zwischen 203 und $212^{\circ}$. Das Rohproduct schmilzt meist einige Grade höher als das Umkrystallisirte. Im Trockenschranke auf $140^{\circ}$ erhitzt, zersetzt sich der Körper allmählich, indem die Verbindung $\mathrm{C}_{7} \mathrm{H}_{8} \mathrm{~N}_{4} \mathrm{O}_{4}$ neben viel nicht krystallisirbaren Substanzen entsteht.

I. $0,2314 \mathrm{~g}$ gaben $0,3400 \mathrm{CO}_{2}$ und $0,0872 \mathrm{H}_{2} \mathrm{O}$.

II. $0,2479 \mathrm{~g} " 0,3684 \mathrm{CO}_{2}, 0,0953 \mathrm{H}_{8} \mathrm{O}$.

III. $0,1699 \mathrm{~g} \quad " \quad 0,2485 \mathrm{CO}_{3} \quad, \quad 0,0718 \mathrm{H}_{2} \mathrm{O}$.

IV. $0,1719 \mathrm{~g}, 34,6 \mathrm{ccm}$ feuchtes Stickgas bei $12^{\circ}$ und $756 \mathrm{~mm}$ Druck.

V. $0,1765 \mathrm{~g} \quad, \quad 33,4 \mathrm{ccm}$ feuchtes Stickgas bei $8^{\circ}$ und $771 \mathrm{~mm}$ Druck.

VI. $0,2727 \mathrm{~g} \quad, \quad 52 \mathrm{ccm}$ feuchtes Stickgas bei $8^{\circ}$ und $777 \mathrm{~mm}$ Druck.

Ber. für $\mathrm{C}_{8} \mathrm{H}_{20} \mathrm{~N}_{8} \mathrm{O}_{3}$

C 39,67

H $\quad 4,13$

N 23,14

Gef.

$\begin{array}{cccccc}\text { I } & \text { II } & \text { III } & \text { IV } & \text { V } & \text { VI } \\ 40,08 & 40,53 & 40,42 & - & - & - \\ 4,19 & 4,27 & 4,69 & - & - & - \\ - & - & - & 23,71 & 23,80 & 23,54\end{array}$

Obwohl die für Kohlenstoff gefundenen Werte durchweg zu hoch sind, lässt sich doch keine andere als die angegebene Formel aus dem Körper berechnen.

Der Körper entsteht in geringer Menge auch beim Erhitzen von Oxymethylhydantoïn mit wenig Wasser bis anf $200^{\circ}$, hauptsächlich wird dabei allerdings der Körper $\mathrm{C}_{7} \mathrm{H}_{8} \mathrm{~N}_{4} \mathrm{O}_{4}$ gebildet.

$$
2 \mathrm{C}_{4} \mathrm{H}_{8} \mathrm{~N}_{2} \mathrm{O}_{3}=\mathrm{C}_{8} \mathrm{H}_{10} \mathrm{~N}_{4} \mathrm{O}_{8}+\mathrm{H}_{2} \mathrm{O} \text {. }
$$

Die Entstehung aus Hydantoïn und Formaldehrd geht nach der Gleichung

$$
2 \mathrm{C}_{3} \mathrm{H}_{4} \mathrm{~N}_{2} \mathrm{O}_{2}+2 \mathrm{CH}_{2} \mathrm{O}=\mathrm{C}_{8} \mathrm{H}_{10} \mathrm{~N}_{4} \mathrm{O}_{5}+\mathrm{H}_{2} \mathrm{O}
$$

vor sich; die Constitution wird vielleicht durch die Formel 


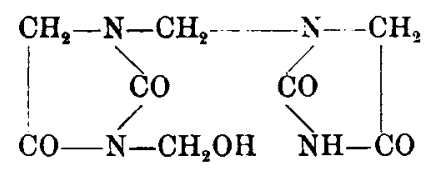

veranschaulicht. Die Bildung aus Oxymethylhydantoïn würde zwar eher für eine unsymmetrische Stellung der Methylengruppe sprechen; die Reaction vollzieht sich aber unter Abspaltung von Formaldehyd, die Formaldehydreste brauchen daher in dem Producte nicht mehr dieselbe Stellung wie im Oxymethylhydantoïn einzunehmen.

$$
\text { Körper } \mathrm{C}_{7} \mathrm{H}_{8} \mathrm{~N}_{4} \mathrm{O}_{4} \text {. }
$$

Die Verbindung $\mathrm{C}_{7} \mathrm{H}_{8} \mathrm{~N}_{4} \mathrm{O}_{4}$ entsteht durch Einwirkung von Formaldehyd anf Hydantoïn in stark salzsaurer Lösung

$$
2 \mathrm{C}_{3} \mathrm{H}_{4} \mathrm{~N}_{2} \mathrm{O}_{2}+\mathrm{CH}_{2} \mathrm{O}=\mathrm{C}_{7} \mathrm{H}_{3} \mathrm{~N}_{4} \mathrm{O}_{4}+\mathrm{H}_{2} \mathrm{O} \text {. }
$$

Die Constitution wird wahrscheinlich durch die Formel

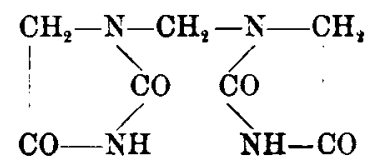

ausgedrückt.

Der Körper bildet sich auch aus Oxymethylhydantoïn unter Austritt von Formaldehyd und Wasser

$$
2 \mathrm{C}_{4} \mathrm{H}_{6} \mathrm{~N}_{2} \mathrm{O}_{8}=\mathrm{C}_{7} \mathrm{H}_{8} \mathrm{~N}_{4} \mathrm{O}_{4}+\mathrm{CH}_{2} \mathrm{O}+\mathrm{H}_{2} \mathrm{O} \text {, }
$$

sowie aus der Verbindung $\mathrm{C}_{8} \mathrm{H}_{10} \mathrm{~N}_{4} \mathrm{O}_{5}$ unter Austritt von Formaldehyd

$$
\mathrm{C}_{8} \mathrm{H}_{10} \mathrm{~N}_{4} \mathrm{O}_{5}=\mathrm{C}_{7} \mathrm{H}_{3} \mathrm{~N}_{4} \mathrm{O}_{4}+\mathrm{CH}_{3} \mathrm{O} \text {. }
$$

$4 \mathrm{~g}$ Hydantoïn werden in einem Gemisch von $5 \mathrm{~g}$ $40 \%$ iger Formaldehydlösung und $2 \mathrm{~g}$ conc. Salzsäure in der Hitze gelöst und dann mit dem gleichen Volum Wasser verdünnt. Im Verlaufe eines Tages krystallisiren $1,0-1,1 \mathrm{~g}$ aus, innerhalb weiterer 10 Tage noch $0,7-0,8 \mathrm{~g}$; der beim Verdunsten hinterbleibende Rest stellt einen nichtkrystallisirenden Syrup dar. Die Ge- 
sammtansbeute an krystallisirtem Product beträgt also 1.8 oder $42 \%$ der berechneten; zuweilen wurden aber unter anscheinend denselben Versuchsbedingungen wesentlich geringere Ausbeuten erhalten.

Aus Oxymethylhydantoïn gewinnt man den Körper durch etwa $3^{1 / 2}$ stündiges Erhitzen auf $120^{\circ}$, bis die Schmelze glasklar geworden ist, und Umkrystallisiren der nach dem Erkalten harten und spröden Masse aus heissem Wasser. Die Ausbeute beträgt etwa $45 \%$ der theoretisch möglichen; die Krystalle, welche sich aus der eingeengten Mutterlauge noch abscheiden, lassen sich nicht mehr von dem zugleich entstandenen dicken Syrup trennen.

Löst man Oxymethylhydantoïn in möglichst wenig heissem Wasser und erhitzt ïber freier Flamme bis $200^{\circ}$, so bleibt nach dem Erkalten eine spröde Masse zurück, aus welcher durch fractionirte Krystallisation aus Wasser die Verbindungen $\mathrm{C}_{7} \mathrm{H}_{8} \mathrm{~N}_{4} \mathrm{O}_{4}$ und $\mathrm{C}_{8} \mathrm{H}_{10} \mathrm{~N}_{4} \mathrm{O}_{5}$ gewonnen werden können; die erstere in einer Ausbeute ron etwa $36 \%$ der berechneten.

Im vortheilhaftesten stellt man den Körper aus der Verbindung $\mathrm{C}_{8} \mathrm{H}_{10} \mathrm{~N}_{4} \mathrm{O}_{5}$ dar, indem man sie in heissem Wasser unter Zusatz von einigen Tropfen Salzsäure löst und zur Krystallisation eindampft. Die Mutterlauge kann man noch ein zweites und drittes Mal in derselben Weise behandeln, bis nur noch syrupförmige Producte zuriickbleiben. Die Ausbeute beträgt etwa $86 \%$ der berechneten.

Auch die bei der Darstellung des Körpers $\mathrm{C}_{8} \mathrm{H}_{20} \mathrm{~N}_{4} \mathrm{O}_{5}$ fallende Mutterlange kann man durch wiederholtes Eindampfen und Krystallisiren auf die Verbindung $\mathrm{C}_{7} \mathrm{H}_{8} \mathrm{~N}_{4} \mathrm{O}_{4}$ verarbeiten.

Ferner kann man sie durch kurzes Kochen der Verbindung $\mathrm{C}_{8} \mathrm{H}_{10} \mathrm{~N}_{4} \mathrm{O}_{5}$ mit conc. Salzsäure darstellen. Sie scheidet sich dann im Verlaufe einiger Tage krystallinisch ab.

Schliesslich entsteht sie neben syrupförmigen Pro- 
ducten aus dem noch $z u$ besprechenden Condensationsproduct $\mathrm{C}_{14} \mathrm{H}_{18} \mathrm{~N}_{6} \mathrm{O}_{9}$, wenn man es eine Stunde lang mit Wasser kocht.

Die nach einer dieser Methoden dargestellte Verbindnng ist in Wasser viel schwerer löslich als der Körper $\mathrm{C}_{8} \mathrm{H}_{10} \mathrm{~N}_{4} \mathrm{O}_{5}$ und verändert sich auch bei anhaltendem Kochen nicht; aus der erkalteten Lösung kommt sie in charakteristischen lanzettförmigen Kryställchen, welche das polarisirte Licht parallel und senkrecht zur Längsrichtung auslöschen. Sie beginnt bei etwa $285^{\circ}$ zu sintern und schmilzt bei $295^{\circ}$ zu einer braunen Flüssigkeit.

I. $0,2204 \mathrm{~g}$ gaben $0,2217 \mathrm{~g} \mathrm{CO}_{2}$ und $0,0804 \mathrm{~g} \mathrm{H}_{q} \mathrm{O}$.

II. $0,2680 \mathrm{~g} \quad, 58,1 \mathrm{ccm}$ feuchtes Stickgas bei $7^{\circ}$ und $757 \mathrm{~min}$ Druck.

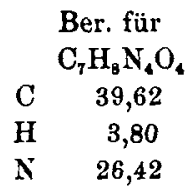

Gef.

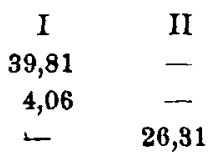

Körper $\mathrm{C}_{14} \mathrm{H}_{18} \mathrm{~N}_{8} \mathrm{O}_{9}$.

$5 \mathrm{~g}$ Hydantoïn werden in $7 \mathrm{~g}$ heisser $40 \%$ iger Formaldehydlösung gelöst, $7 \mathrm{~g}$ conc. Salzsäure zugesetzt und kurze Zeit zum Sieden erhitzt. Die Salzsäure darf erst nach völliger Lösung des Hydantoïns zugesetzt werden, sonst löst es sich erst nach längerem Kochen und es entstehen viel syrupförmige Nebenproducte, deren Bildung sich übrigens niemals ganz vermeiden lässt. Nach einigen Tagen scheiden sich Nester von derben Krystallen aus, die bei $180^{\circ}$ schmelzen. Durch Umkrystallisiren aus heissem Wasser erhält man feine farblose Krystalle. Der Schmelzpunkt liegt bei $183-188^{\circ}$. Die Ausbeute an Rohproduct beträgt im Mittel $4 \mathrm{~g}$ oder $\bar{\jmath} 6 \%$ der berechneten Menge. Beim Umkrystallisiren geht etwa der vierte Theil verloren. 
I. $0,2294 \mathrm{~g}$ gaben $0,3396 \mathrm{~g} \mathrm{CO}_{2}$ und $0,1000 \mathrm{~g} \mathrm{H}_{2} \mathrm{O}$.

II. $0,2701 \mathrm{~g}, 0,4025 \mathrm{~g} \mathrm{CO}_{2}, 0,1244 \mathrm{~g} \mathrm{H}_{2} \mathrm{O}$.

III. $0,2216 \mathrm{~g} \quad, 40,6 \mathrm{ccm}$ feuchtes Stickgas bei $21^{\circ}$ und $764 \mathrm{~mm}$ Druck.

IV. $0,2941 \mathrm{~g}$ gaben 50,4 ccm feuchtes Stickgas bei $15^{\circ}$ und $766 \mathrm{~mm}$ Druck.

V. $0,2357 \mathrm{~g}$ gaben $41,18 \mathrm{ccm}$ feuchtes Stickgas bei $18^{\circ}$ und $757 \mathrm{~mm}$ Druck.

Ber. für

$\begin{array}{lccccccc} & \mathrm{C}_{9} \mathrm{H}_{12} \mathrm{~N}_{4} \mathrm{O}_{8} & \mathrm{C}_{14} \mathrm{H}_{18} \mathrm{~N}_{8} \mathrm{O}_{8} & \text { I } & \text { II } & \text { III } & \text { IV } & \text { V } \\ \mathrm{C} & 39,67 & 40,54 & 40,36 & 40,63 & - & - & - \\ \mathrm{H} & 4,44 & 4,38 & 4,84 & 5,11 & - & - & - \\ \mathrm{N} & 20,63 & 20,33 & - & - & 20,87 & 20,13 & 20,31\end{array}$

Die gefundenen Werthe stimmen am besten mit den für die Formel $\mathrm{C}_{14} \mathrm{H}_{18} \mathrm{~N}_{8} \mathrm{O}_{8}$ berechneten überein, weichen aber auch von den für $\mathrm{C}_{9} \mathrm{H}_{12} \mathrm{~N}_{4} \mathrm{O}_{6}$ berechneten nicht sehr weit ab.

$$
\begin{aligned}
& 3 \mathrm{C}_{8} \mathrm{H}_{4} \mathrm{~N}_{2} \mathrm{O}_{2}+5 \mathrm{CH}_{2} \mathrm{O}=\mathrm{C}_{14} \mathrm{H}_{18} \mathrm{~N}_{6} \mathrm{O}_{0}+2 \mathrm{H}_{2} \mathrm{O} \\
& 2 \mathrm{C}_{8} \mathrm{H}_{4} \mathrm{~N}_{2} \mathrm{O}_{2}+3 \mathrm{CH}_{2} \mathrm{O}=\mathrm{C}_{9} \mathrm{H}_{12} \mathrm{~N}_{4} \mathrm{O}_{6}+\mathrm{H}_{2} \mathrm{O} .
\end{aligned}
$$

Die Bestimmung des Gefrierpunktes der Lösung in Wasser (Constante $=18,5$ ) ergab folgende Werthe für das Molekulargewicht.

\begin{tabular}{cc|c|c}
\hline $\begin{array}{c}\text { Procentgehalt der } \\
\text { Lösung }\end{array}$ & $\begin{array}{c}\text { Gefrierpunkts } \\
\text { erniedrigung }\end{array}$ & $\begin{array}{c}\text { Berechnetes } \\
\text { Molekulargewicht }\end{array}$ \\
\hline I. 0,8525 & a) 0,089 & 175,2 \\
b) 1,171 & a) 0,090 & 185,2 \\
III. 1,422 & b) 0,118 & 187,9 \\
IV. 1,461 & a) 0,140 & 192,1 \\
b) 0,140 & a) 0,141 & 197,6 \\
b) 1,688 & a) 0,141 & Mittel $: 187,6$
\end{tabular}

Für die Formel $\mathrm{C}_{14} \mathrm{H}_{18} \mathrm{~N}_{6} \mathrm{O}_{9}$ ist das Molekulargewicht 414, für die Formel $\mathrm{C}_{9} \mathrm{H}_{12} \mathrm{~N}_{4} \mathrm{O}_{6} 272$ berechnet. Der gefundene $W$ erth entspricht nahezu der Hälfte des ersteren: 
207. Wenn man versucht, die Formel $\mathrm{C}_{14} \mathrm{H}_{18} \mathrm{~N}_{6} \mathrm{O}_{0}$ structurchemisch zu deuten, so muss man eine Molekel Formaldehyd als Krystallformaldehyd annehmen (Formel I) und das Ergebniss der Molekulargewichtsbestimmung könnte so erklärt werden, dass bei der Lösung Dissociation unter Abspaltung des Krystallformaldehyds stattfindet. Man könnte aber auch an eine Structur denken, wie sie etwa durch die Formel II veranschaulicht wird, die Molekulargewichtsbestimmung würde dann auf electrolytische Dissociation deuten.

I

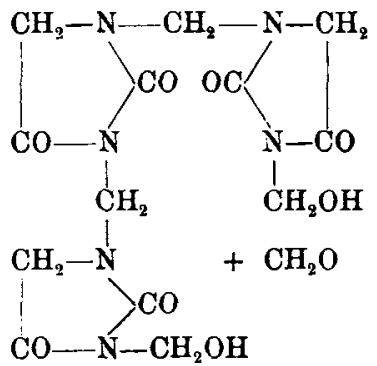

II

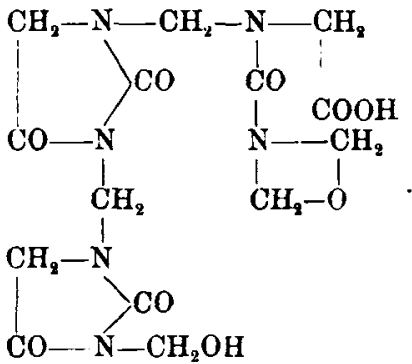

Bei längerem Erhitzen im Trockenschrank beginnt der Körper bereits bei $130^{\circ}$ sich zu zersetzen; durch längeres Kochen mit Wasser oder Alkohol lässt er sich unter starker Entwickelung von Formaldehyd vollständig in die Verbindung $\mathrm{C}_{7} \mathrm{H}_{3} \mathrm{~N}_{4} \mathrm{O}_{4}$ überführen.

$$
2 \mathrm{C}_{14} \mathrm{H}_{18} \mathrm{~N}_{6} \mathrm{O}_{8}+\mathrm{H}_{2} \mathrm{O}=3 \mathrm{C}_{7} \mathrm{H}_{8} \mathrm{~N}_{4} \mathrm{O}_{4}+7 \mathrm{CH}_{8} \mathrm{O} \text {. }
$$

\section{Amorphe Condensationsproducte.}

Ans der Mutterlauge von der Darstellung des Körpers $\mathrm{C}_{14} \mathrm{H}_{3} \mathrm{~N}_{8} \mathrm{O}_{8}$ fällt auf Zusatz von absolutem 4 kohol eine amorphe Substanz, welche nach mehrfachem Auskochen mit Alkohol als harter Kuchen zurückbleibt. Der Schmelzpunkt der über Schwefelsäure getrockneten Substanz liegt bei $83-85^{\circ}$; an der Luft zieht sie Wasser an und geht in einen zähen Syrup über; in heissem Wasser löst sie sich und wird durch Alkohol wieder ge- 


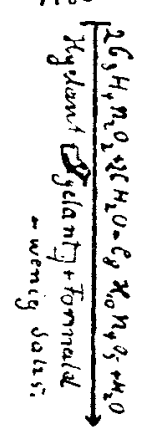

$G_{14} H_{18} n_{6} O_{g} S_{m} P_{183-188^{\circ}}$
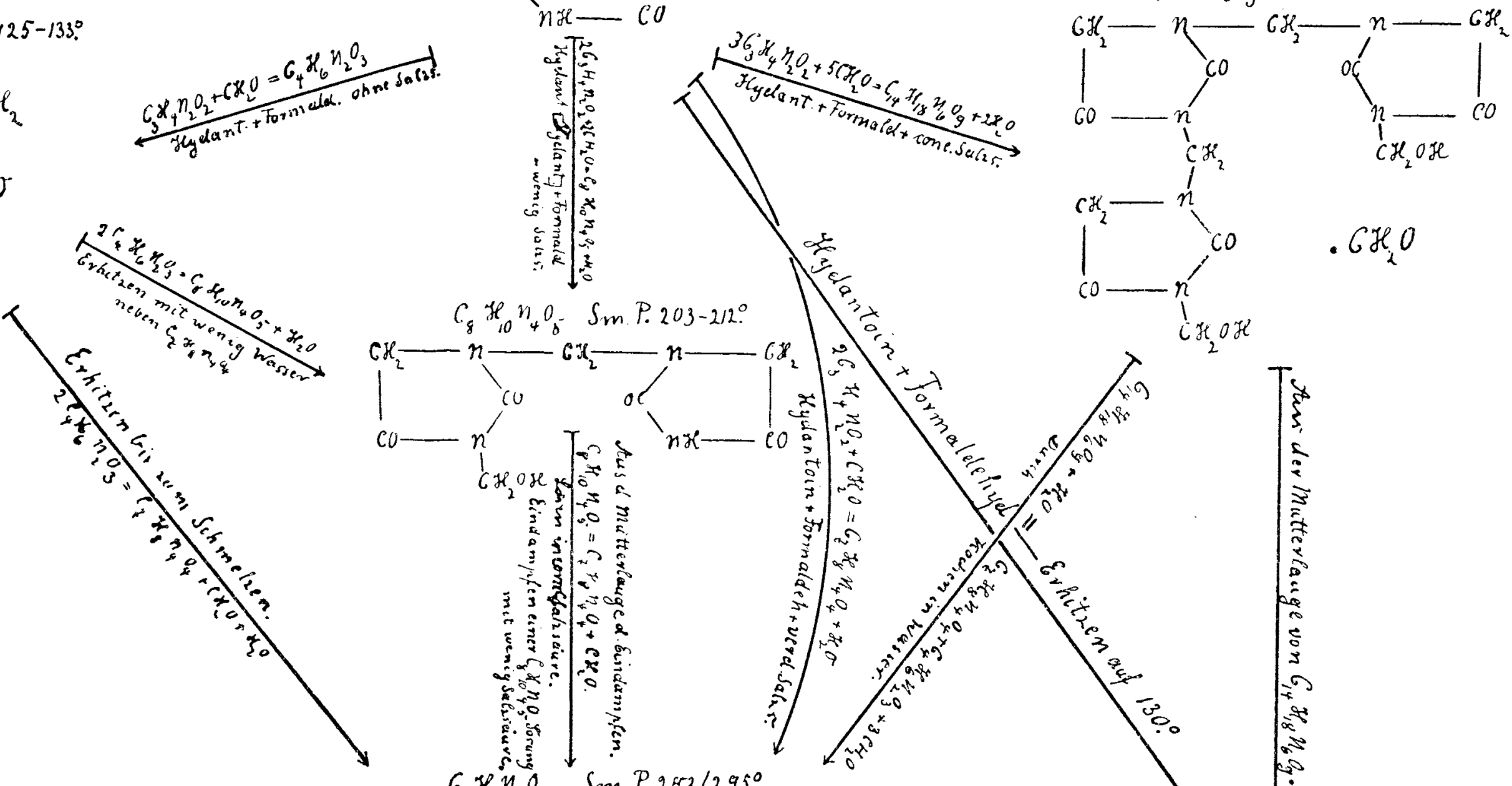

$n_{8} n_{4} n_{0} S_{m} P_{203} 212^{\circ}$
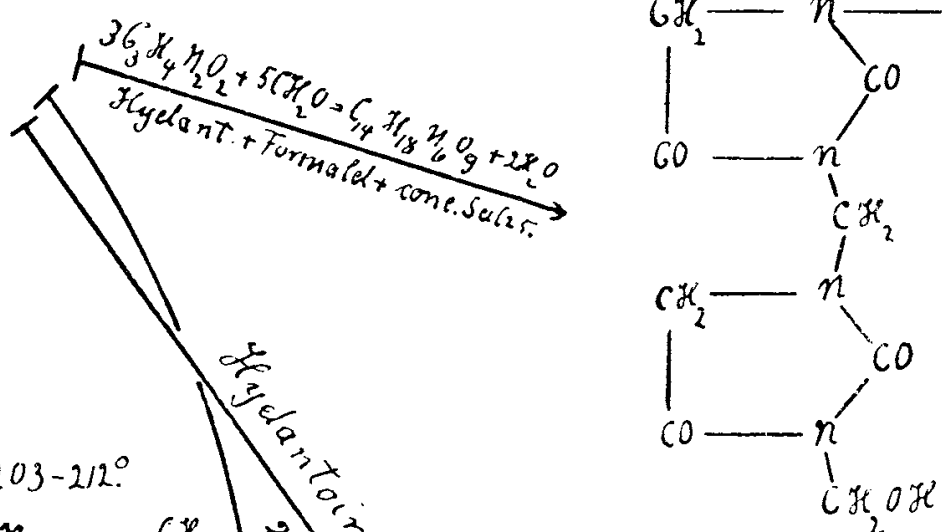

$.6 H_{2} \mathrm{O}$

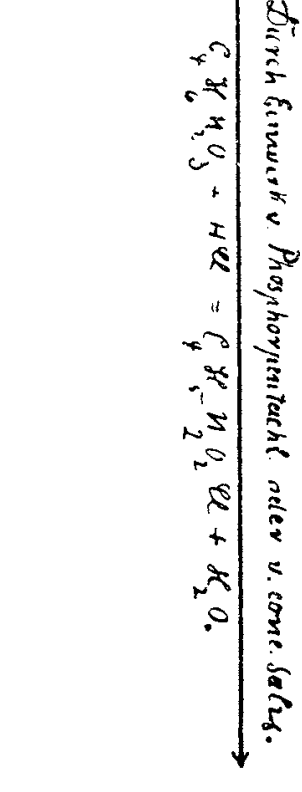
roto

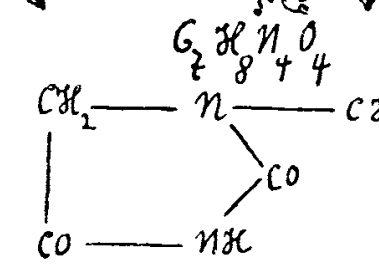

$S_{m}$. P. 283/295.
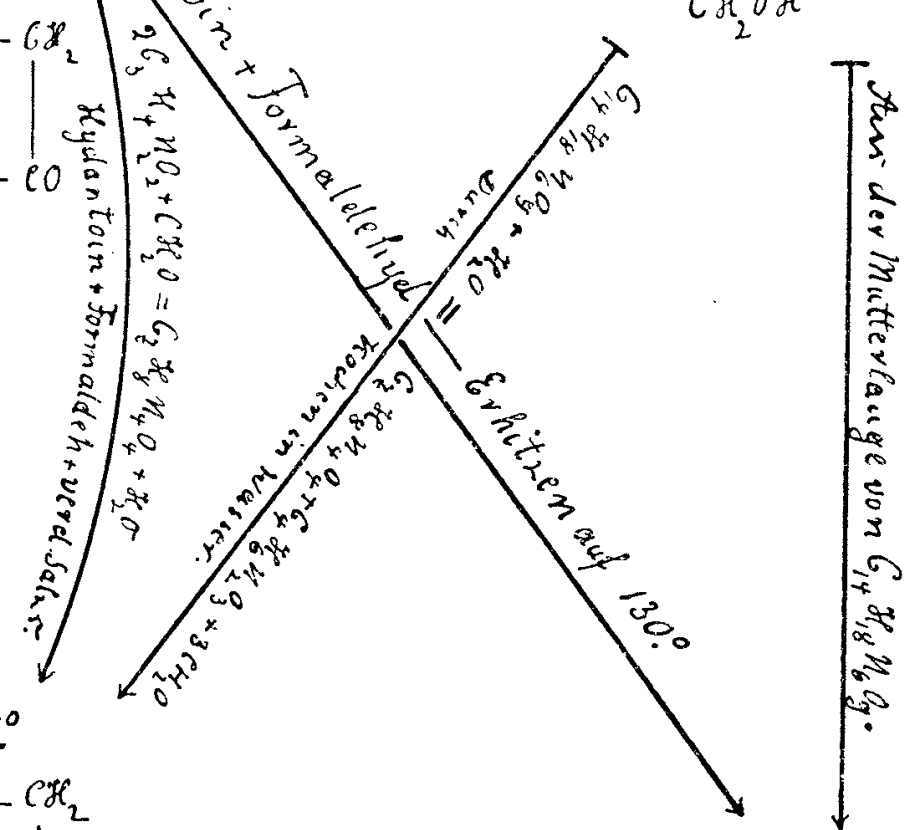

Amorphes Kondensetionsprodukt. Sm P 83-85. 
fällt. Der Schmelzpankt ändert sich dabei nicht. In conc. Salzsäure löst sich der Körper sehr leicht auf, bei nicht zu starkem Verdünnen mit Wasser wird er wieder abgeschieden. In krystallisirten Zustand konnte er nicht übergeführt werden. Man kann die Snbstanz auch erhalten, wenn man Hydantoïn (5 Gewichtsth.) und 40\% ige Formaldehydlösung (7 Gewichtsth.) 2 Stunden im Einschmelzrohr auf $125-130^{\circ}$ erhitzt, zur Hälfte eindampft und mit Alkohol behandelt.

Auch aus Oxymethylhydantoïn bildet sie sich beim Erhitzen mit Formaldehyd auf $130^{\circ}$ oder beim Lösen in conc. Jodwasserstoffsäure. Vermuthlich enthalten auch die Syrupe, welche bei der Darstellung der übrigen Condensationsproducte als Nebenproducte entstehen, dieselbe Substanz. Die Analyse ergab Werthe, welche mit den für die Formel $\mathrm{C}_{14} \mathrm{H}_{18} \mathrm{~N}_{8} \mathrm{O}_{2}$ übereinstimmen. $\mathrm{Da}$ es aber zweifelhaft ist, ob die Substanz einheitlich ist, ausserdem die Kenntnis des Molekulargewichts fehlt und die für eine ganze Reihe hochmolekularer Condensationsproducte berechneten Zahlen sich sehr wenig unterscheiden, wäre die tufstellung einer bestimmten Formel verfrüht.

I. $0,1633 \mathrm{~g}$ gaben $0,2453 \mathrm{CO}_{3}$ und $0,0755 \mathrm{H}_{2} \mathrm{O}$.

II. $0,2051 \mathrm{~g} \# \quad 0,3085 \mathrm{CO}_{2}, 0,0954 \mathrm{H}_{2} \mathrm{O}$.

III. $0,3077 \mathrm{~g}, 52,8 \mathrm{ccm}$ feuchtes Stickgas bei $7^{\circ}$ und $759 \mathrm{~mm}$ Druck.

IV. $0,2513 \mathrm{~g}$ gaben $44,4 \mathrm{~cm}$ feuchtes Stickgas bei $8^{0}$ und $755 \mathrm{~mm}$ Druck.

Ber. für

$\begin{array}{cccccc} & \mathrm{C}_{16} \mathrm{H}_{18} \mathrm{~N}_{8} \mathrm{O}_{8} & \text { I } & \text { II } & \text { III } & \text { IV } \\ \mathrm{C} & 40,54 & 40,69 & 41,01 & - & - \\ \mathrm{H} & 4,38 & 5,13 & 5,16 & - & - \\ \mathrm{N} & 20,33 & - & - & 20,05 & 20,78\end{array}$

Die genetischen Beziehungen der verschiedenen Condensationsprodncte sind in der beigegebenen Tabelle übersichtlich zusammengestellt. 